Document downloaded from:

http://hdl.handle.net/10251/51051

This paper must be cited as:

Crespo Amorós, JE.; Parres, F.; Peydro, MA.; Navarro Vidal, R. (2013). Study of rheological, Thermal and Mechanical Behavior of Reprocessed Polyamide 6. Polymer Engineering and Science. 53(4):679-688.

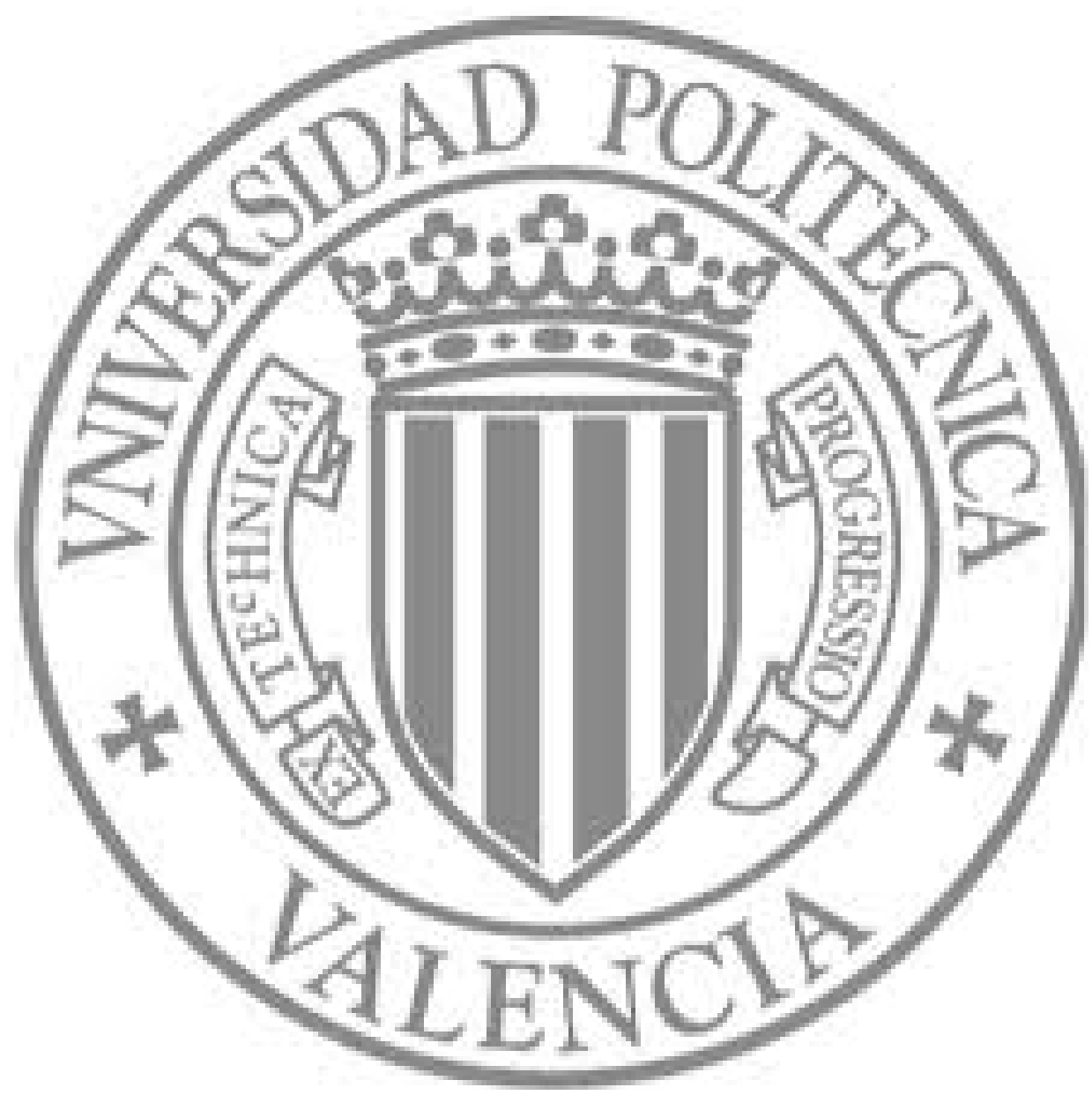

The final publication is available at

http://dx.doi.org/10.1002/pen.23307

Copyright Wiley-Blackwell 


\title{
STUDY OF RHEOLOGICAL, THERMAL AND MECHANICAL BEHAVIOR OF REPROCESSED POLYAMIDE 6
}

\author{
J. E. Crespo; F. Parres; M. A. Peydró; R. Navarro \\ Department of Mechanical and Materials Engineering \\ Universitat Politècnica de València \\ Plaza Ferrándiz y Carbonell, s/n 03801 Alcoy - España \\ Telephone and fax: +34966528570
}

E-mail: jocream@dimm.upv.es*, raunavi@dimm.upv.es, mpeydro@mcm.upv.es,

fraparga@dimm.upv.es

\begin{abstract}
The effect of reprocessing Polyamide 6 (PA6) has been studied in this paper. To simulate recycled PA, we reprocessed virgin PA through 5 cycles. The PA 6 has undergone mechanical, thermal and rheological characterization after the various cycles of reprocessing in order to evaluate the corresponding properties and correlate them with the number of cycles undergone. In order to widen our injection simulation analysis by computer (CAE: Computer Aided Engineering) of these new materials, it was necessary to determine the viscosity using a mathematical model; in this case the Cross-WLF, to determine the relevant parameters.

Our results show that tensile strength, elongation at break and hardness remain practically constant, while the charpy impact decreases as the number of reprocessing cycles increases. The effects of reprocessing on the material may decrease the rheological properties; specifically the viscosity of the material decreases with increasing processing cycles. The thermal properties are also influenced with the reprocessed material. The crystallinity increases and the degradation reaction will be advanced to increase the reprocessing cycle.
\end{abstract}

\section{Keywords}

Rheology, Material testing, Reprocessing, Thermal properties, Injection.

\section{INTRODUCTION}

Polyamide 6 (PA6) has been extensively applied in many fields because it possesses a range of excellent properties, and its consumption will continue growing in the future. Therefore, how to recycle and reuse effectively, as well as restrain degradation during processing will become increasingly important subjects for polymer scientists and engineers. Consequently, the influence of reprocessing operations on the characterization of PA6 must be known in advance. 
The problems which arise in the recovery of polymer materials are basically caused by the variations that occur in their properties, due to either thermal degradation or the presence of impurities. Many studies have been carried out on the degradation of polymers. Su [1] analyzed the influence of the reprocessing cycles on the mechanical properties, rheological properties and changes in structure of PA6. Su's work is relevant to our work because he carried out a study of mechanical and rheological properties. We can find interesting studies on reprocessing, [1], but we do not consider that reprocessing the material a huge number of times is very useful because other studies show that the loss of properties occurs in the first 5 reprocessing cycles. In previous work on reprocessed material, the authors have generally only studied the Melt Flow Index (MFI) as a rheological property and have not studied the real viscosity of the material, and thus, our calculations on the real viscosity obtained in this work is a valid contribution to exiting work on these materials.

Moreover, some studies have found that reprocessing PA6 shows interesting behavior, [2], since the material's viscosity increases as the material is reprocessed. This surprising behavior can be explained by the fact that different moisture contents of PA6 can cause contrary behaviour. [3] showed that there is a decrease in viscosities at high humidity content and increasing viscosity for very dry samples.

Other studies conducted on the influence of moisture in PA6, $[4,5]$ where work with industrial waste consisting of glass fiber reinforced PA6 composites was dried for 3, 6 and 9 hours before reprocessing, also showed that the reprocessing of this type of composite was viable, because satisfactory physical-mechanical properties are attained thereafter, which make them suitable for several applications where these properties are desirable. The drying period of $6 \mathrm{~h}$ was the minimum necessary time for the reprocessed material to present a good visual appearance. The author also showed, by means of TGA analysis, that the drying period has a marked influence on the final moisture content of PA 6 composites.

Other studies were made in order to know how many times it is possible to recycle nylon-6 without significant loss of the physical-mechanical properties [6]. The nylon-6 was recycled 10 times, and up to the eighth cycle the properties of the material did not suffer any change. Changes of 10-15\% in the properties occurred with nylon-6 with 10 injection cycles of virgin material [6]. This author also shows the darkening effect that takes place after each cycle. Optical and electronic microscopy was used to evaluate the morphology. Like other authors, he only studied the MFI as a rheological property and not the real viscosity of the material, so our study broadens the understanding gained from previous publications. 
Various authors have investigated the mechanical and rheological properties of recycled material (derived from industrial production waste) and filled (with $20 \%$ glass beads and $10 \%$ glass fiber) PA6 [7]. Studied this as a function of the number reprocessing operations and of the percentage of recycled material added to the virgin material. This work shows that the properties of the recycled material remained inferior to the virgin, and the best combination of both appeared to be the mixture with $30 \mathrm{wt}$. \% recycled material.

We can also find studies on the mixture of polymers with the aim of improving properties of the recovered material, [8]. In his study of the influence of polyethylene (PE) on recycled polyethylene terephtalate (PET) analyzed, among other things, mechanical and rheological properties. His work is of interest to us because he uses the Cross equation to calculate viscosity, although he only uses a die (L/D $10 \mathrm{~mm}$ ) so that the viscosity calculated is apparent rather than exact. In his study, he obtained values of viscosity that got lower as the percentage of PE in the mixtures was increased. It is also interesting because he also studied in more detail the shear rate interval between 5.000 and $10.000 \mathrm{~s}^{-1}$. The study of this interval is important as these values most closely match the conditions to which the material is subject during the injection process.

One of the fundamental properties to consider when studying polymer processing is the rheological behavior. Understanding this parameter is vital in order to carry out a correct extrusion or injection of the polymer. The real viscosity of a polymer is obtained using a capillary rheometer along with the Bagley and Rabinowitsc corrections.

Some injection processing simulations, such as Moldflow Plastics Inside 6.2 ®, use values of the parameters from the Cross-WLF model, [9, 10]. These experiments were carried out to reproduce the rheological behavior of the materials during the injection process. There are studies which apply these models in the reprocessing of thermoplastic materials, [11-13]. These studies are interesting because the authors calculated the Cross dependent parameters of the mixtures and show how variations in these affect viscosity. At the same time, he showed that increasing the temperature has a greater influence on the degradation of thermoplastic than increases in the shear rate.

In other studies, $[12,13]$, the authors also determine a Material Processability Index (MPI) for three mixtures of thermoplastics with a different Melt Flow Index (MFI). The authors also calculated the Cross parameters for each mixture and use the methodology for the design of experiments (DOE). The variables reused in the DOE were the variables from injection processing (filling time, melt temperature, coolant temperature, packing pressure level and 
filling-packing switch-over). For all of this, computer simulation of the injection process to determine the mixture content of thermoplastics was used.

The objective of this present work is to study the rheological behaviour of PA6 from virgin material through to the fifth reprocessing cycle. Other objectives of this present work include to mechanically and thermally characterize the material after the various cycles of reprocessing in order to evaluate its corresponding properties and correlate them with the number of cycles undergone. Finally, a further objective of this present work is to determine the parameters of the Cross-WLF model, both for virgin and recycled material in order to incorporate them into plastic injection simulations. 


\section{EXPERIMENTAL}

\subsection{Materials}

To develop this study, we used PA6 Heramid S Neg 233 supplied by the company Radici Plastic ${ }^{\circledR}$.

\subsection{Methods and measurements.}

The rheological analysis of all the materials was done using a Thermo Haake Rheoflixer MT® capillary rheometer. The temperature used in the rheometer was $240{ }^{\circ} \mathrm{C}$, which is well within the recommended range of the manufacturer. The shearing speeds used in the study were in the range 100 to $10,000 \mathrm{~s}^{-1}$. The rheometer was equipped with three dies, all with a diameter of $1 \mathrm{~mm}$ and with $L / D$ ratios of 10,20 and 30 . The tests were carried out in compliance with ISO 11443. The viscosity values for each die correspond with the average of the five experimental tests.

For the injection, we used a Meteor 270/75 injector supplied by Mateu \& Solé® (Barcelona, Spain) at $240{ }^{\circ} \mathrm{C}$ injection temperature. We carried out five injection cycles (R1, R2, R3, R4 and R5) beginning with virgin PA (V). Figure 1 shows the outline of processing study conducted in the PA6 Virgin.

\section{Figure 1.}

For the mechanical characterization, we used a universal traction machine ELIB 30 (S.A.E. Ibertest, Madrid, Spain) in compliance with ISO 527. A $50 \mathrm{~mm} / \mathrm{min}$ cross-head speed was used to determine the elongation at break and tensile strength from the tensile graph using a load cell of $5 \mathrm{kN}$. The Shore D Hardness was measured using a Baxlo Durometer (Baxlo, Barcelona, Spain) according to ISO 868.

For the calculation of the cristallinity we used a differential scanning calorimeter (DSC) Mettler Toledo, S.A.E. ${ }^{\circledR}$, model $821 \mathrm{e}$, a type of power compensation DSC in compliance with ISO 11357-1 y 11357-4. The thermogravimetric study was carried out using equipment of TGA / SDTA Mettler Toledo, mod. 851.

The impact strength was determined with a charpy impact machine (J. Bot S.A, Barcelona, Spain) according to ISO 179.

The values of all mechanical parameters were calculated as averages of five to seven samples for each cycle. 


\section{METHODOLOGY FOR VISCOSITY CALCULATION}

Capillary rheology allows us to obtain the apparent viscosity $\left(\eta_{a p}\right)$ of the polymer under test without any difficulty using the following equations and a constant temperature:

$$
\begin{gathered}
\eta_{a p}=\frac{\tau_{a p}}{\gamma_{a p}} \\
\tau_{a p}=\frac{p \cdot D}{4 \cdot L}
\end{gathered}
$$

Where: $\tau_{a p}$ is the apparent shear stress $[\mathrm{Pa}], \square \gamma_{a p}$ is the apparent shear rate $\left[\mathrm{s}^{-1}\right], p$ is the test pressure in pascals, $D$ is the diameter of the die in $\mathrm{mm}$ and $L$ is the length of the die in $\mathrm{mm}$.

Figures $2 \mathrm{a}, 2 \mathrm{~b}$ and $2 \mathrm{c}$ show the pressures obtained using dies of $L / D$ 10, 20 and 30 for the materials studied. We can see that by increasing the shear rate we increase the pressure regardless of the die used. These values are then used to calculate viscosity.

Figure 2.

The passage of melted material from a greater to a lesser diameter causes over-pressure which slightly alters the results obtained directly from the capillary rheometer. This variation can be corrected using Bagley's correction. The melted polymers are fluid and not Newtonian and the equations used to calculate viscosity must also be adjusted. One of the most commonly used corrections is that of Rabinowitsch, with which it is possible to obtain the true shear rate at the capillary wall. These correction methods are common to calculate the real viscosity, $[11,13]$.

\subsection{Bagley's correction.}

The application of this method required that we use the polymer at a particular temperature and with three dies with different length / diameter ratios $(L / D=10, L / D=20, L / D=30)$, to obtain the pressure in each die at different shear rate $\left(100,200,500,1000,2000,5000,10000\left[\mathrm{~s}^{-1}\right]\right)$. Figure 3 shows the linear trend for the pressure as a function of the $L / D$ ratio of the dies used and in which we can see the adjustment produced for $L / D=0$. The Bagley method corrects the over-pressure related to an apparent increase in die length. Thus, with Equation (3), we calculated the true shear stress $\tau[\mathrm{Pa}]$, where $p$ is the pressure obtained by the adjustment and we used the corrected $L / D$ ratio, $(L / D)_{c}$ which is the $L / D$ distance at which the pressure is zero. 


$$
\tau=\frac{P}{4\left[(L / D)+(L / D)_{c}\right]}
$$

\section{Figure 3.}

\subsection{Rabinowitsc's correction.}

Rabinowitsc's correction allows us to determine the true shear rate $\gamma \square\left[\mathrm{s}^{-1}\right]$ using Equation (4.) To obtain the data with greater precision, it is necessary to represent graphically the true shear stress $\log (\tau)$ against the true shear rate $\log (\gamma)$, Figure 3, and adjust the points of cubic Equation (5). In Equation (4), for the data of the $\log \tau, \log \tau_{\text {adjust }}$ adjusted for the curve of Equation (5). In Figure 2, we can see that the equation used is perfectly adjusted to the points calculated for the virgin material and die $L / D 10$. This was also calculated for $L / D 20$ y 30 .

$$
\gamma=\frac{\gamma_{a p}}{4} \cdot\left(3+\frac{d \log \gamma_{a p}}{d \log \tau}\right)
$$

Where $\gamma_{a p}\left[\mathrm{~s}^{-1}\right]$ is the apparent shear rate.

$$
y=a_{0}+a_{1} \cdot x+a_{2} \cdot x^{2}+a_{3} \cdot x^{3}
$$

\section{Figure 4.}

\subsection{Calculation of viscosity.}

The viscosity was calculated with the quotient between shear stress and shear rate, as seen in Equation (6). As with the calculation of viscosity, three dies were used, we also obtained three viscosity curves for each material, Figure 5. From these three curves, one single curve was calculated as a final result.

$$
\eta=\frac{\tau}{\gamma}
$$

\section{Figure 5.}




\subsection{Determination of dependent parameters from the Cross-WLF model; determination of viscosity of the material.}

The viscosity value obtained is the result of the complete process at a constant temperature. Viscosity varies with temperature, Equation (7) and Equation (8). The Cross model [9] allows us to calculate the viscosity of the material at whichever temperature beginning with a viscosity curve of a given temperature.

$$
\eta=\frac{\eta_{0}}{1+\left(\frac{\eta_{0}}{\tau^{*}} \cdot \gamma\right)^{1-n}}
$$

Where $\eta_{0}[\mathrm{~Pa} \mathrm{~s}]$ is material viscosity under zero-shear-rate conditions, $\tau^{*}[\mathrm{~Pa}]$ is the model constant that shows the shear stress rate, from which the pseudoplastic behavior of the material starts, $\mathrm{n}[-]$ is the model constant which symbolizes the pseudoplastic behavior slope of the material as: $(1-n)$.

To determine the viscosity of the material with zero shear, we used the Williams-Landel-Ferry Equation (8).

$$
\eta_{0}=D_{1} \cdot e^{\frac{-A_{1} \cdot(T-\tilde{T})}{A_{2}+(T-\tilde{T})}}, \text { si T } \geq \tilde{T}
$$

$$
\begin{aligned}
& A_{2}=\tilde{A}_{2}+D_{3} \cdot p \\
& \tilde{T}=D_{2}+D_{3} \cdot p
\end{aligned}
$$

where $\tilde{T}[\mathrm{~K}]$ is the glass transition temperature of the material depending on the pressure. $D_{2}$ $[\mathrm{K}]$ is the model constant which registers the glass transition temperature of the material at atmospheric pressure. $D_{3}[\mathrm{~K} / \mathrm{Pa}]$ is the model constant which symbolizes the variation of the glass transition temperature of the material, according to the pressure. $D_{1}[\mathrm{~Pa} \mathrm{~s}]$ is the model constant which registers the material viscosity, under zero shear rate conditions, at material glass transition temperature and at atmospheric pressure. $A_{l}[-]$ is the model constant that shows the temperature dependence of material glass transition temperature under zero shear rate 
conditions. $\tilde{A}_{2}[\mathrm{~K}]$ is a model parameter that depends on the type of material that has been considered.

\subsection{Calculation method of the Cross WLF model.}

After obtaining the three viscosity curves, Figure 5, it was necessary to calculate the collective viscosity of the material, for which two methods were used. The first was the chi squared test and the second was squared minimums. After the study, we found the chi squared method adjusts better. The determination of viscosity was carried out using the independent parameters of the Cross WLF model.

The independent parameters of the Cross model are those which only depend on the material and which were obtained from Moldflow $6.1{ }^{\circledR}$ simulation data and from the DSC. $\tilde{A}_{2}=51.60$ K. $D_{2}=\mathrm{T}_{\mathrm{g}}=50^{\circ} \mathrm{C}=323.15 \mathrm{~K}$.

$$
D_{3}=\frac{\partial T_{g}}{\partial p}=0 \mathrm{~K} / \mathrm{Pa}
$$

The dependent parameters of the Cross model which must be calculated are: $n[-] ; \tau^{*}[\mathrm{~Pa}] ; D_{l}$ $[\mathrm{Pa} \cdot \mathrm{s}] ; A_{1}[-]$

In order to calculate these parameters we used the MathCad $2001 \AA$ program for the interaction and separation of data from the three dies. The equation for this calculation can be seen in Equation (10).

ChiCuadrado $(\mathrm{D} 1, \mathrm{~A} 1, \tau, \mathrm{n}$, datos $):=\sum_{\mathrm{i}=0}^{\operatorname{rows}(\text { datos })-1}\left[\frac{\left(\text { datos }_{\mathrm{i}, 2}-\eta\left(\text { datos }_{\mathrm{i}, 0}, \text { datos }_{\mathrm{i}, 1}, \mathrm{D} 1, \mathrm{~A} 1, \tau, \mathrm{n}\right)\right)^{2}}{\eta\left(\text { datos }_{\mathrm{i}, 0}, \text { datos }_{\mathrm{i}, 1}, \mathrm{D} 1, \mathrm{~A} 1, \tau, \mathrm{n}\right)}\right]$

Once the parameters were obtained, they were introduced into the data base of Moldflow Plastics Inside 6.2 in order to then obtain the material viscosity graphics. The Cross-WLF method allows us to obtain a single real viscosity curve starting from the experimental curves by type of die. This method allows us to predict the real viscosity of the material for any temperature. Figure 6 shows the real viscosity for the virgin material using Cross-WLF method for two temperatures different from the $240{ }^{\circ} \mathrm{C}$ experimental temperature used in this work.

Figure 6. 


\section{RESULS AND DISCUSSION}

\subsection{Analysis of mechanical properties.}

The mechanical properties of any material are fundamental for its use in any particular application. The traction test is extremely important because it allows us to understand properties such as tensile strength and elongation at break.

Thermal degradation as a consequence of recycling processes causes changes in the internal structure of the polymer and these changes in turn cause variations in its mechanical properties. In the study carried out on PA6, Table 1, showing the values of tensile strength with the injection cycles, reveals a slightly decreasing lineal evolution in the values. The same behavior is observed for the values of elongation at break as a function of injection cycles, showing a slight drop in value. However, hardness values show a loss in the first two cycles, and then remain constant.

Regarding the performance of the PA6 for impact strength, the values show a progressive decrease with increasing cycles of reprocessing. The impact strength drastically decreases from $45.09 \mathrm{~J} / \mathrm{m} 2$ (the Virgin PA6) to $28.61 \mathrm{~J} / \mathrm{m} 2$ (the 5 th processed PA6), as seen in Table 1 . The reduction of impact resistance with reprocessing cycles seems to correlate with the behavior of molecular chain scission. Shorter molecular chain and broader chain length distribution result in poor chain entanglements, resulting in loss of strength for the reprocessed PA6. Other factors such as contamination particles and gel particles that produce the defective sites in PA6 matrix should be must be considered when accounting for the reduction in impact resistance. This behavior has been studied previously and similar results and conclusions were obtained, [1].

\section{Table 1.}

\subsection{Analysis of rheological properties.}

Figure 5 shows the behavior of the perfect virgin material (V) and the first recycled (R1) to obtain a single viscosity curve, regardless of the die used. In reprocessed R2, R3, R4 and R5, there is a dispersion of results depending on the die used. These curves should be as close as possible to minimize errors. Therefore, we use the Cross-WLF model to unify the results into a single viscosity curve.

Table 2 shows the values of the Cross-WLF model and the viscosity of the material at zero shear rates. These values are very important in enabling us to carry out an accurate injection simulation using data on reprocessed material, given that no other data base exists. 
Table 2.

The variation in the value $n$, Figure $7 \mathrm{a}$, (a constant of the model represented as (1-n) which describes the pseudoplastic behavior of the material) diminishes slightly with each new reprocessing cycle. This slight increase in the slope of the curve causes a slight reduction in viscosity under the same conditions of zero shear rates.

The variation of the value $\tau^{*}$, Figure $7 b$, which shows the shear stress rate from which the pseudoplastic behavior of the material begins, increases slightly with each cycle. This behavior causes the viscosity curve to move to the right, causing a slight increase in viscosity, considered to be a negative aspect of the material's behavior.

The parameter $D_{l}$ (a constant of the model which shows the viscosity of the material in zero shear rate conditions, and at the transition temperature and atmospheric pressure) and the parameter $A_{l}$ (a constant of the model which shows the sensitivity of the viscosity at zero shear rate at the temperature) are not represented as the model developed with MathCad 2001 ® can give the same viscosity values for different pairs of $D_{l}$ and $A_{l}$.

The variation in the value $\eta_{0}$, viscosity of the material at zero shear rates was calculated with the Williams-Landel-Ferry Equation (7). As can be seen in Figure 7c, there is a slight drop in this value.

Figure 7.

Of the three parameters $n, \tau^{*} \mathrm{y} \eta_{0}$, it is the last which makes the viscosity of the material diminish slightly as the number of cycles increases, above all at low shear rates up to $200 \mathrm{~s}^{-1}$. The reduction in $n$ (which increases the slope of the curve) and the increase in $\tau^{*}$ (which moves the curve to the right) cause the curves to cross and join in the area of high shear rate $\left(1,000 \mathrm{~s}^{-1}\right.$ and above). This behavior can be seen in the graphics of Figure 8 and Figure 9.

\section{Figure 8.}

Viscosity is a property that is extremely sensitive to variations in molecular weight. Viscosity decreases due to degradation of the material (breaks in the polymer chains which then become shorter), as the material is repeatedly reprocessed (extruded and pelletized). This behavior is similar to that obtained by other authors, [1], when reprocessing PA6. The viscosity of PA6 
diminishes with repeated cycles, although the final data are not comparable as only the Melt Flow Index was calculated in these works.

Moreover, other studies on reprocessing PA6, [2], show the complete opposite behavior. The viscosity of the material increases with reprocessing. [3] explained that different moisture content of PA6 can cause contrary behavior. This author shows decreasing viscosities at high humidity content and increasing viscosity for very dry samples. This is exactly what one would expect from the equilibrium polycondensation reaction, Equation (11).

$$
-\mathrm{COOH}+-\mathrm{NH}_{2} \leftrightarrow-\mathrm{NHCO}-+\mathrm{H}_{2} \mathrm{O}(1)
$$

The Le Chatelier-Braun principle predicts the effect of a change in conditions in a chemical equilibrium. Higher water contents shift the equilibrium reaction to the left side of Equation (1), thereby reducing the water content and causing chain scission, i.e., reducing the molecular weight. On the other hand, at very low water content the reaction is shifted to the right side. The condensation reaction transforms two end groups into one peptide bond and one water molecule, respectively, thereby increasing the humidity and molecular weight of the sample. This indicates that at a water content of about $0.12 \%$, the viscosity, and by implication the molecular weight of the PA6 sample, remain constant for different residence times in the rheometer at $260{ }^{\circ} \mathrm{C},[3]$.

The equilibrium humidity concentration for constant molecular weight will depend on the concentration of reactive end groups of the polyamide and the operation parameters of the Karl Fischer measurement (e.g., temperature) and the capillary rheometer (e.g., the time and temperature needed to fill the cylinder).

In capillary rheometer flow the material is under high hydrostatic pressure and the water will be soluble in the melt due to the high hydrostatic pressure and no gas bubbles will be formed. Thus, the water molecules remain dissolved and may act as a plasticizer.

In the capillary rheometer, the sample humidity will partly be lost due to evaporation during the sample filling step. As a consequence of the high temperature and hydrostatic pressure, the water that remains in the sample after the cylinder is filled and closed by the barrel will be dissolved in the polyamide melt, such that the flow curves of viscosity $v s$ shear rate will reflect both the change in molecular weight and the plasticizing effect of the dissolved water, increasing the viscosity and increasing the drying time of the sample. 
The study of shear rate in the interval 5,000 and $10,000 \mathrm{~s}^{-1}$ is of great importance, as these values are reached by the plastic during the injection process, [8]. Figure 9 shows the fall in viscosity at these shear rates with an increase in number of cycles.

\section{Figure 9.}

\subsection{Analysis of thermal properties.}

The thermal properties studied as a function of reprocessing cycles, are the crystallinity effect and degradation in the material. The crystallinity is influenced by increasing reprocessing cycles, Table 3, under both isothermal and non isothermal conditions.

\section{Table 3.}

The increase in crystallinity in the reprocessed PA6 versus the virgin PA6 is mainly due to three factors, [14]: (1) Faster crystallization may arise from increased nucleation due to the presence of impurities incorporated in the matrix during processing. (2) Memory effects associated with thermal and mechanical stress histories that remain present in the sample after annealing in the melt may also lead to an increased rate of crystallization. (3) Generation of low molecular weight chains during processing, i.e. through degradation, may facilitate increased polymer chain mobility and lead to faster crystallization. Consequently, the significant increase in crystallization rate from the virgin to the first processed PA6 should be due to memory effects induced by injection moulding, [1].

The thermogravimetric study shows the weight loss that occurs in the PA6 in terms of processing cycles. It also shows information on the degradation reaction of the material and its inflection point. Figure 10 shows the curves obtained for different processing cycles of the PA6.

\section{Figure 10.}

Table 4 shows the results of the study thermogravimetric of PA6. There are small differences in temperature between the reprocessed cycles and the virgin material, but we saw that the degradation reaction produced in the material starts in the earlier reprocessing cycles. This is due to breakage of chains produced by the memory effect during the injection process. 
Table 4.

\section{CONCLUSIONS}

We studied the effects of reprocessing on PA6 material. Our study was centred on the three most important properties of the material: mechanical resistance, viscosity and thermal properties. Results regarding the mechanical properties do not show a big difference, except that the impact resistance shows a significant decrease compared to virgin material. Tensile strength decreases by around $1.42 \%$, lengthening at break is reduced by $1 \%$, hardness is reduced by $1.5 \%$ while charpy impact strength decreases by around $36.5 \%$.

As already commented, this behavior of reduction of impact resistance with reprocessing cycles seems to be caused by molecular chain scission.

Concerning the rheological behavior, viscosity diminishes with each reprocessing cycle. At the fifth cycle, at zero shear rate the viscosity falls by around $72 \%$. This effect is caused by the reduction in the molecular weight caused by degradation of the material due to breaks in the polymer chains, which become shorter as the material is repeatedly reprocessed. Furthermore, as already mentioned the effect of moisture in the polymer makes this work as a plasticizer and further reduce the viscosity. However, in injection moulding processes using shear rates of 5000 to $10000 \mathrm{~s}^{-1}$ viscosity falls by 28 and $23 \%$ respectively. This drop in viscosity is beneficial in plastic injection as less injection pressure can achieve the same results as virgin material, but it also means that as the material is more fluid, more defective pieces are generated (appearance of flash).

As the ultimate objective is to recover the original properties of virgin material, the solution is simply to reduce the injection temperature from 230 to $225^{\circ} \mathrm{C}$. Thus we achieve an energy saving (representing lower environmental impact) as well as optimum re-use of recycled material instead of virgin material, further reducing the impact on the environment.

DSC thermal analysis reveals several differences in crystalline behavior for the virgin and the processed PA6. The significant differences between the virgin sample and the processed ones should be attributed to memory effects induced by injection moulding. Moreover, chain scission resulting from reprocessing operations increases chain mobility and consequently promotes the development of smaller and imperfect crystallite as well as increases the crystallization rate. The break in the chains is also reflected in the results obtained in the thermogravimetric study. The degradation reaction starts at lower temperatures and consequently the inflection point decreases with reprocessing cycles. 


\section{REFERENCES}

[1] Su K.H., Lin J.H., Lin C.C.: Influence of reprocessing on the mechanical properties and structure of polyamide 6. Journal of Materials Processing Technology, 192, 532-538 (2007).

[2] Bonardi A., Cilloni R., Paganuzzi V., Grizzuti N.: Effects of degree of recycling on the rheology and processability of thermoplastic polymers. Journal Of Polymer Engineering, 23, 79-94 (2003).

[3] Dijkstra D.J.: Guidelines for Rheological Characterization of Polyamide Melts. Pure and Applied Chemistry, 81, 339-349 (2009).

[4] Pedroso A.G., Mei L.H.I., Agnelli J.A.M., Rosa D.S.: Properties that characterize the propagation of cracks of recycled glass fiber reinforced polyamide 6. Polymer Testing, 18, 211-215 (1999).

[5] Pedroso A.G., Mei L.H.I., Agnelli J.A.M., Rosa D.S.: The influence of the drying process time on the final properties of recycled glass fiber reinforced polyamide 6 . Polymer Testing, 21, 229-232 (2002).

[6] Lozano-Gonzalez J., Rodriguez-Hernandez T., Los Santos E., Villalpando-Olmos J.: Physical-mechanical properties and morphological study on nylon-6 recycling by injection molding. Journal of Applied Polymer Science, 76, 851-858 (2000).

[7] Maspoch M.L., Ferrando H.E., Velasco J.I.: Characterisation of filled and recycled PA6. Macromolecular Symposia, 194, 295-303 (2003).

[8] Navarro R., Ferrandiz S., Lopez J., Segui V.J.: The influence of polyethylene in the mechanical recycling of polyethylene terephtalate. Journal of Materials Processing Technology, 195, 110-116 (2008).

[9] Cross M.M.: Rheology of Non-Newtonian Fluids - a New Flow Equation for Pseudoplastic Systems. Journal of Colloid Science, 20, 417-437 (1965).

[10] Williams M.L., Landel R.F., Ferry J.D.: Mechanical Properties of Substances of High Molecular Weight. The Temperature Dependence of Relaxation Mechanisms in Amorphous Polymers and Other Glass-Forming Liquids. Journal of the American Chemical Society, 77, 3701-3707 (1955).

[11] Boronat T., Segui V.J., Peydro M.A., Reig M.J.: Influence of temperature and shear rate on the rheology and processability of reprocessed ABS in injection molding process. Journal of Materials Processing Technology, 209, 2735-2745 (2009).

[12] Reig M.J., Segui V.J., Ferrandiz S., Zamanillo J.D.: An evaluation of processability by injection molding of $\mathrm{ABS} / \mathrm{PC}$ blends obtained from recycled materials. Journal of Polymer Engineering, 27, 29-54 (2007). 
[13] Reig M.J., Segui V.J., Zamanillo J.D.: Rheological behavior modeling of recycled ABS/PC blends applied to injection molding process. Journal of Polymer Engineering, 25, 435-457 (2005).

[14] Fornes T.D., Paul D.R.: Crystallization behavior of nylon 6 nanocomposites. Polymer, 44, 3945-3961 (2003). 


\section{Legends of figures}

Figure 1. Diagram of the processing study of virgin PA6.

Figure 2. Pressures obtained for the virgin material and reprocessed materials for different dies: a) (L/D 10), b) (L/D 20) and c) (L/D 30).

Figure 3. Bagley's correction for the virgin material.

Figure 4. Rabinowitsc's correction for virgin material and a die $L / D 10$.

Figure 5. Graphs of viscosity versus shear rate of virgin and reprocessed material PA6.

Figure 6. Real Viscosity of virgin material using the Cross-WLF method at 230, 240 and 250 ${ }^{\circ} \mathrm{C}$.

Figure 7. Dependant parameters of the Cross-WLF model versus processing cycles.

Figure 8. Viscosity of virgin and reprocessed material PA6 at $240{ }^{\circ} \mathrm{C}$.

Figure 9. Viscosity of materials versus number of cycles at shear rate 5.000 and $10000 \mathrm{~s}^{-1}$.

Figure 10. Variation of weight loss in PA6 versus processing cycles. 


\section{$\underline{\text { Legends of tables }}$}

Table 1. Mechanical properties of material virgin and reprocessed PA6.

Table 2. Dependent parameters of the Cross-WLF model for PA6.

Table 3. Variation of crystallinity compared to reprocessing of the PA6.

Table 4. Variation of the inflection point between virgin and reprocessed material. 


\section{Figures}

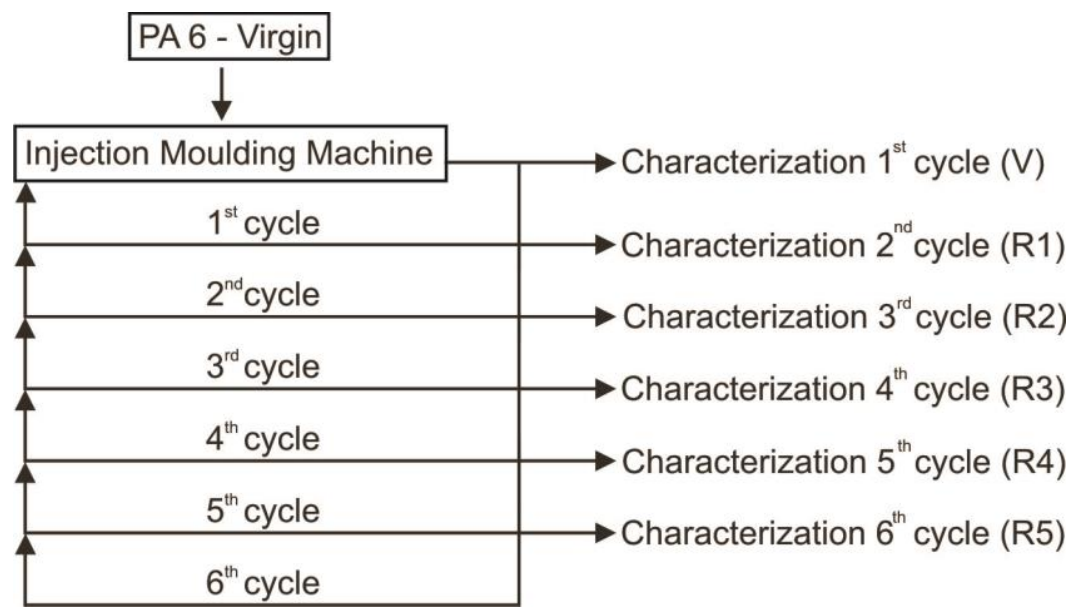

Figure 1. Diagram of the processing study of virgin PA6.

a (L/D 10)

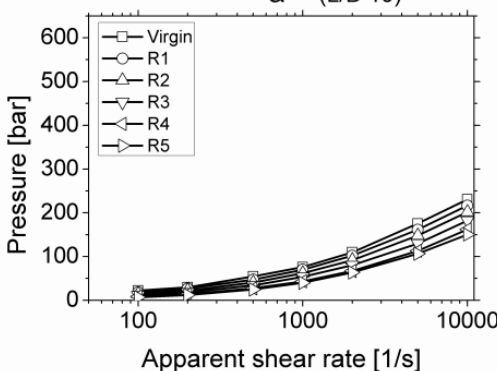

b (L/D 20)

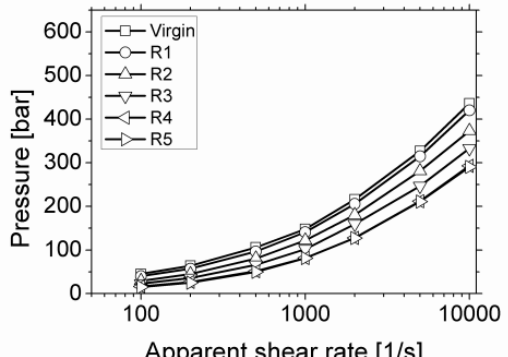

C (L/D 30)

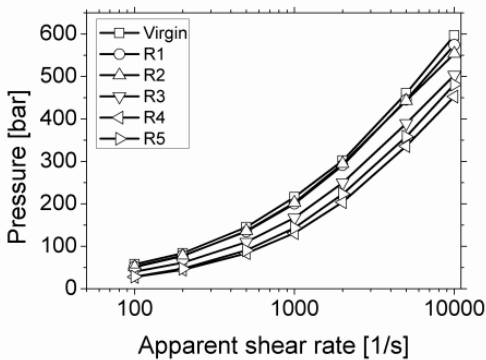

Figure 2. Pressures obtained for the virgin material and reprocessed materials for different dies:

a) $(L / D 10)$, b) (L/D 20) and c) (L/D 30). 


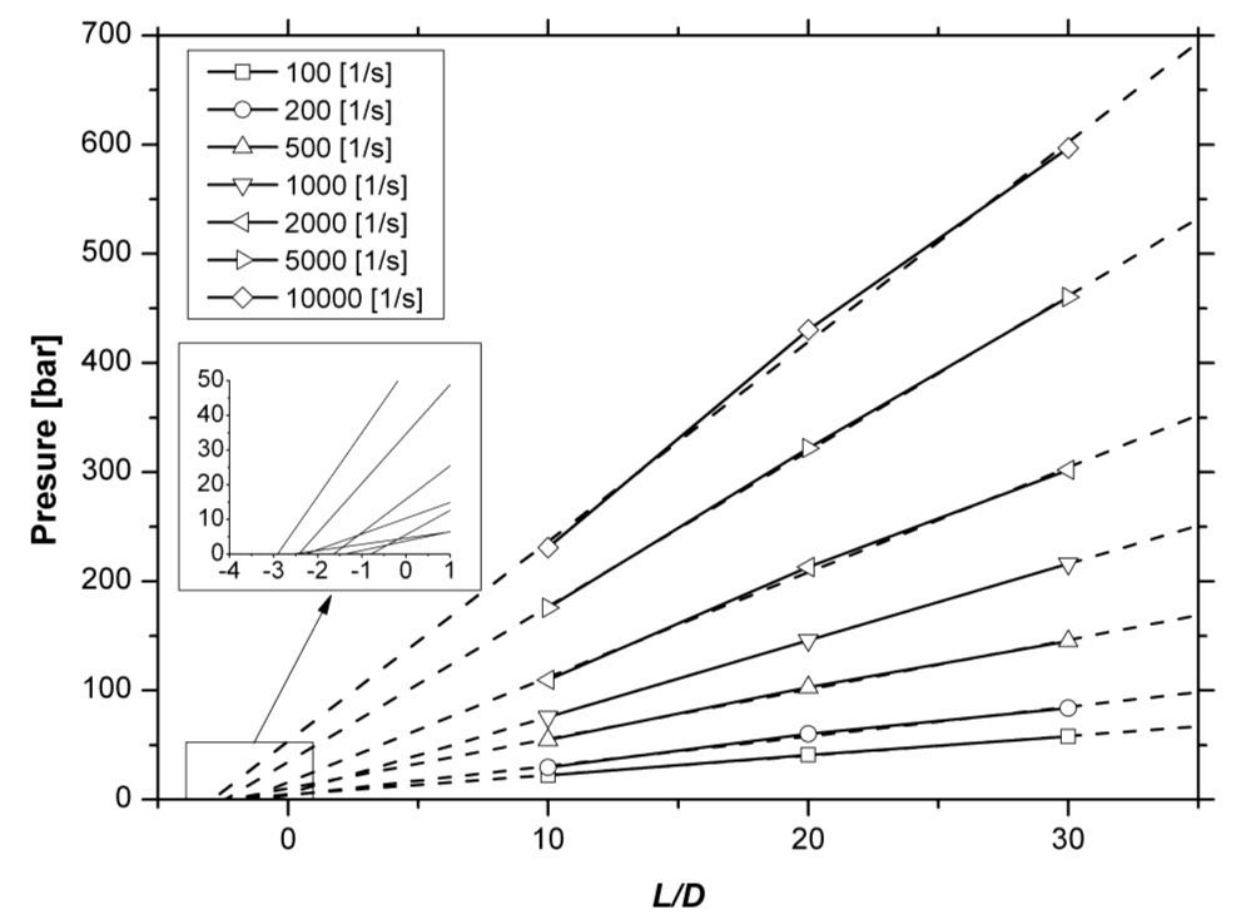

Figure 3. Bagley's correction for the virgin material.

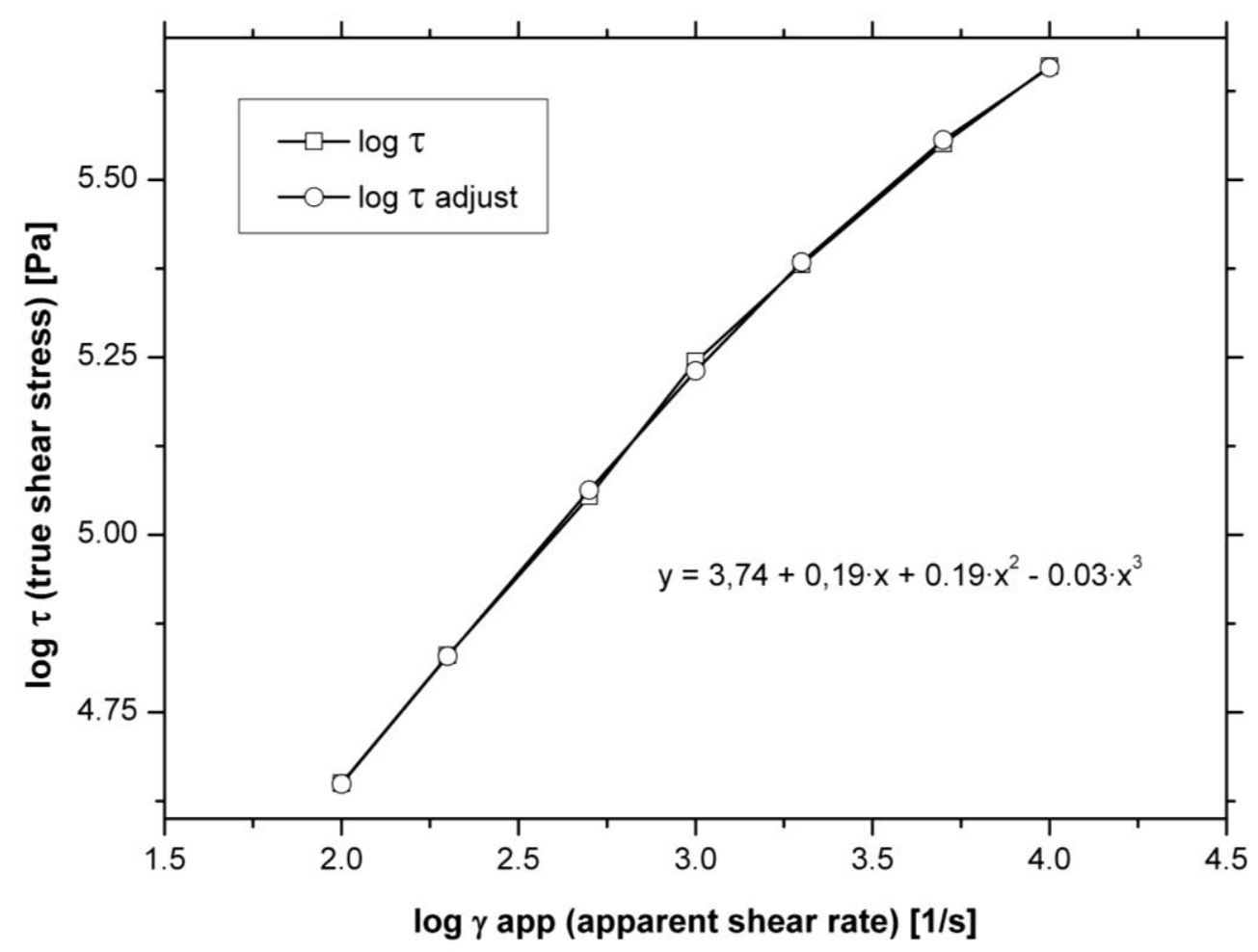

Figure 4. Rabinowitsc's correction for virgin material and a die $L / D 10$. 


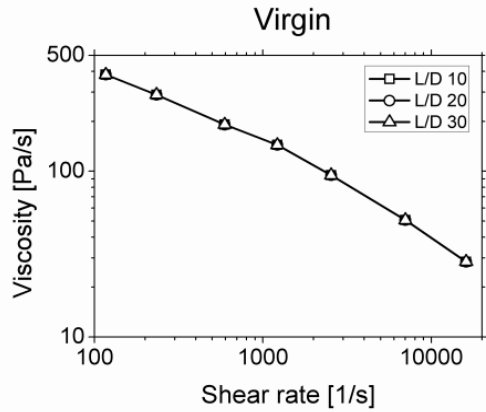

R3

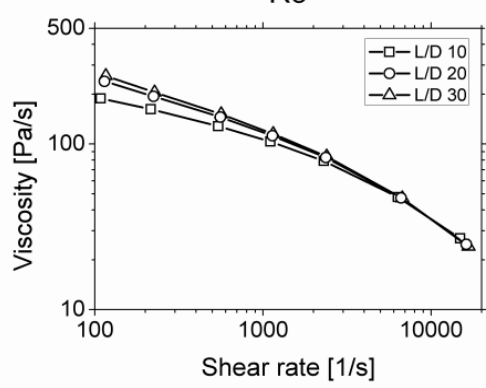

R1

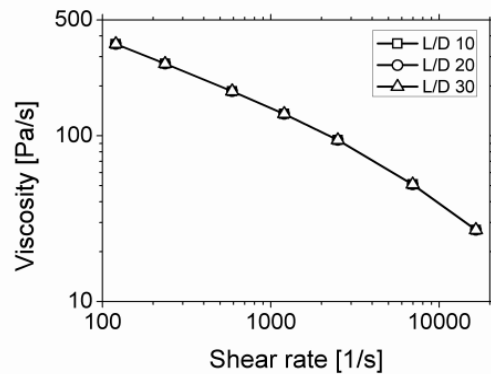

R4

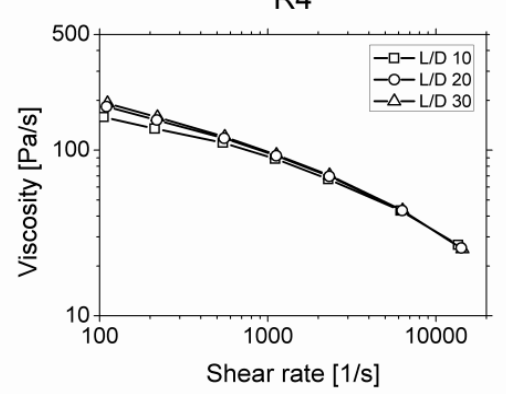

R2

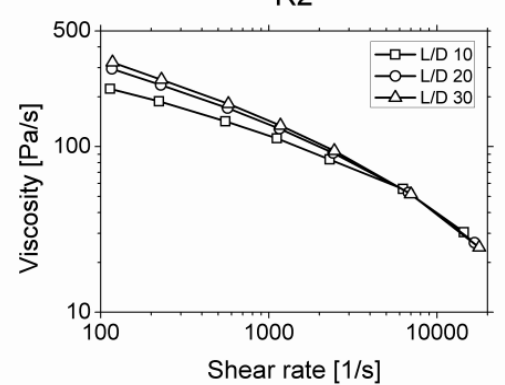

R5

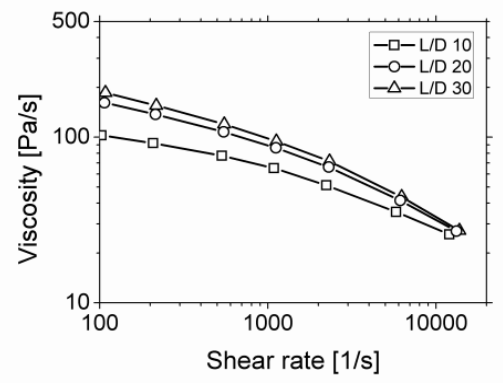

Figure 5. Graphs of viscosity versus shear rate of virgin and reprocessed material PA6.

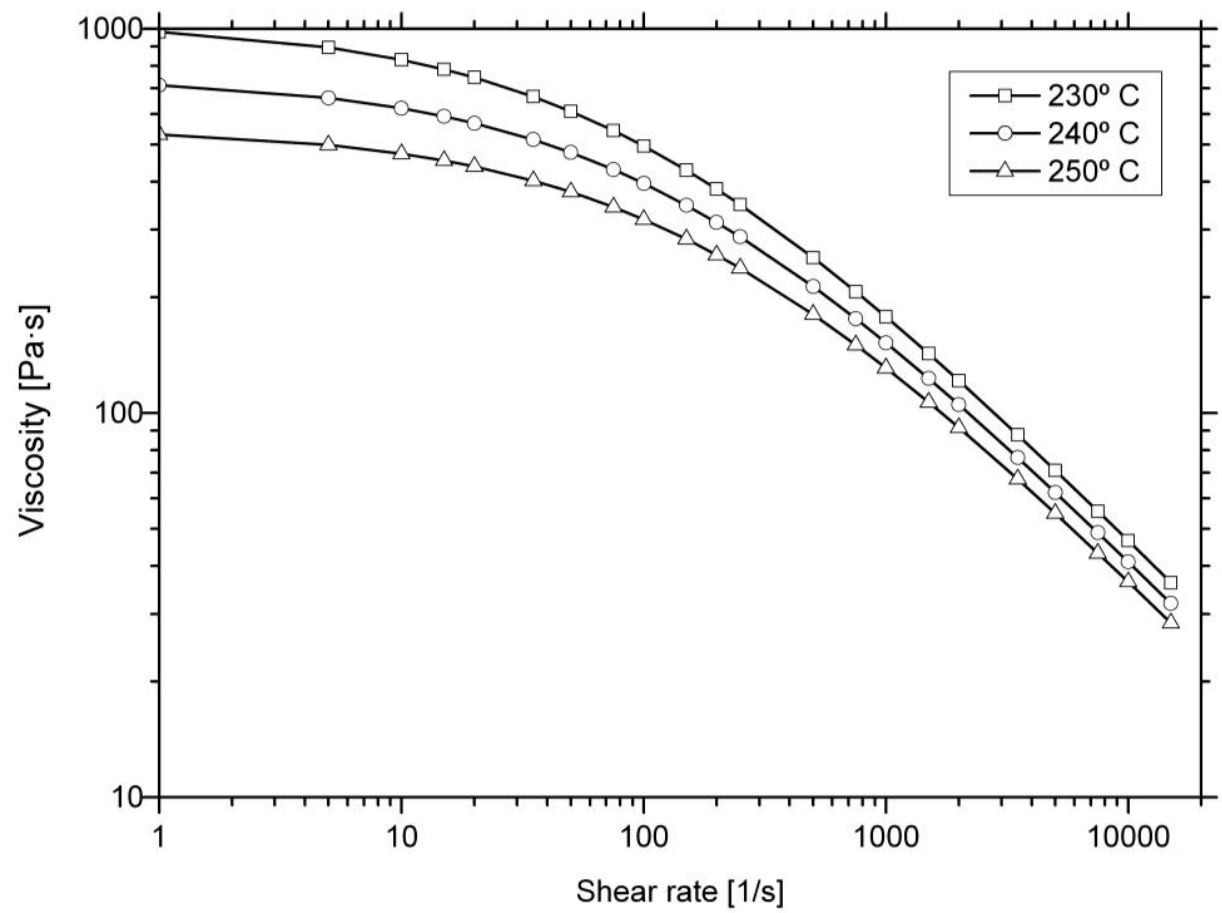

Figure 6. Real Viscosity of virgin material using the Cross-WLF method at 230, 240 and 250 ${ }^{\circ} \mathrm{C}$ 

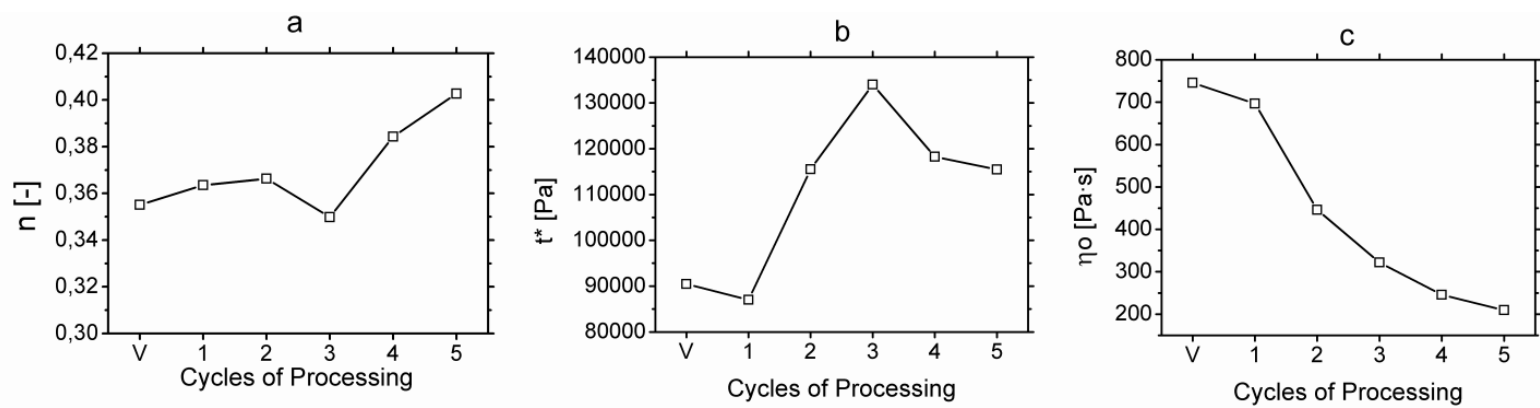

Figure 7. Dependant parameters of the Cross-WLF model versus processing cycles.

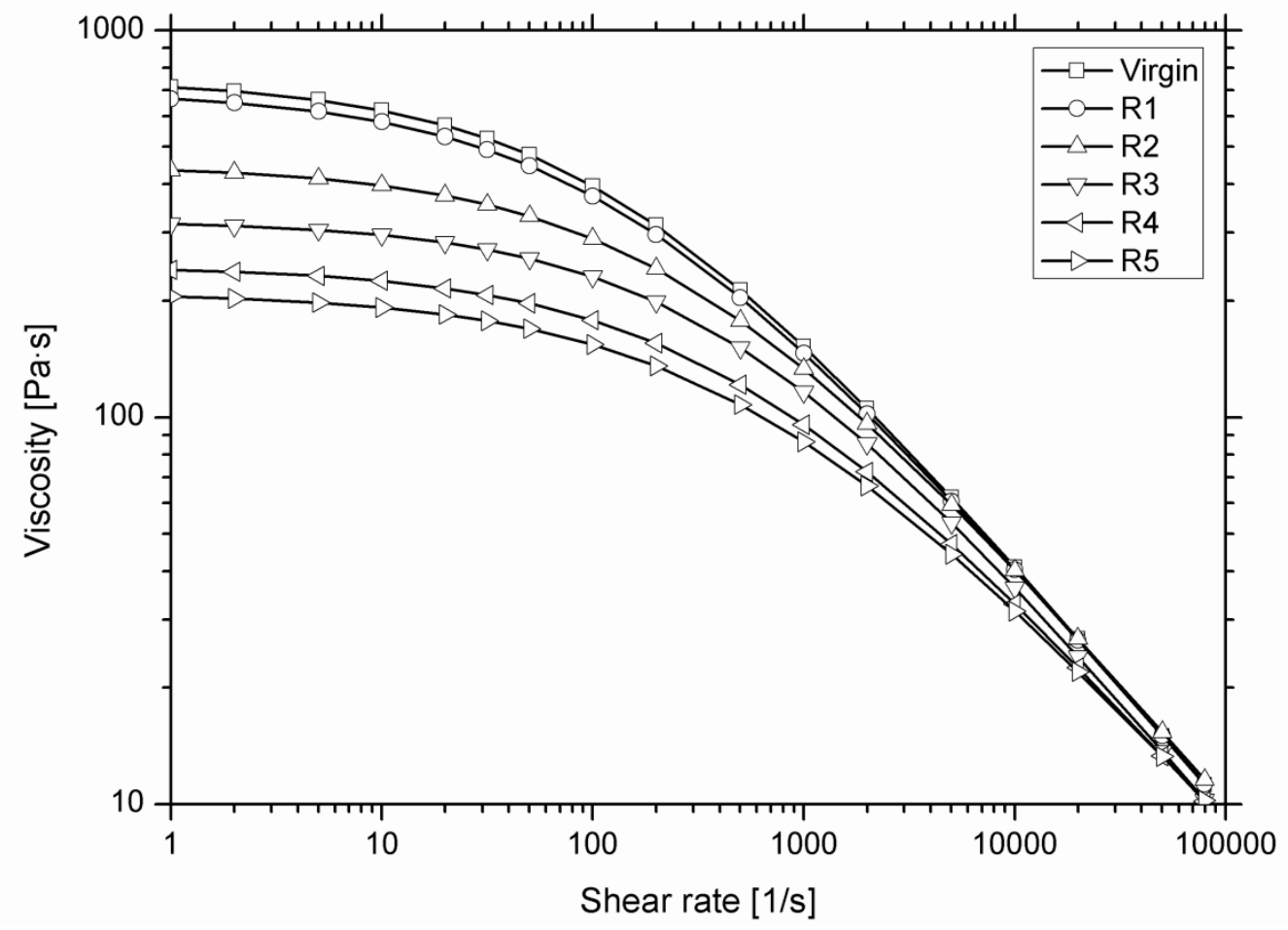

Figure 8. Viscosity of virgin and reprocessed material PA6 at $240{ }^{\circ} \mathrm{C}$. 


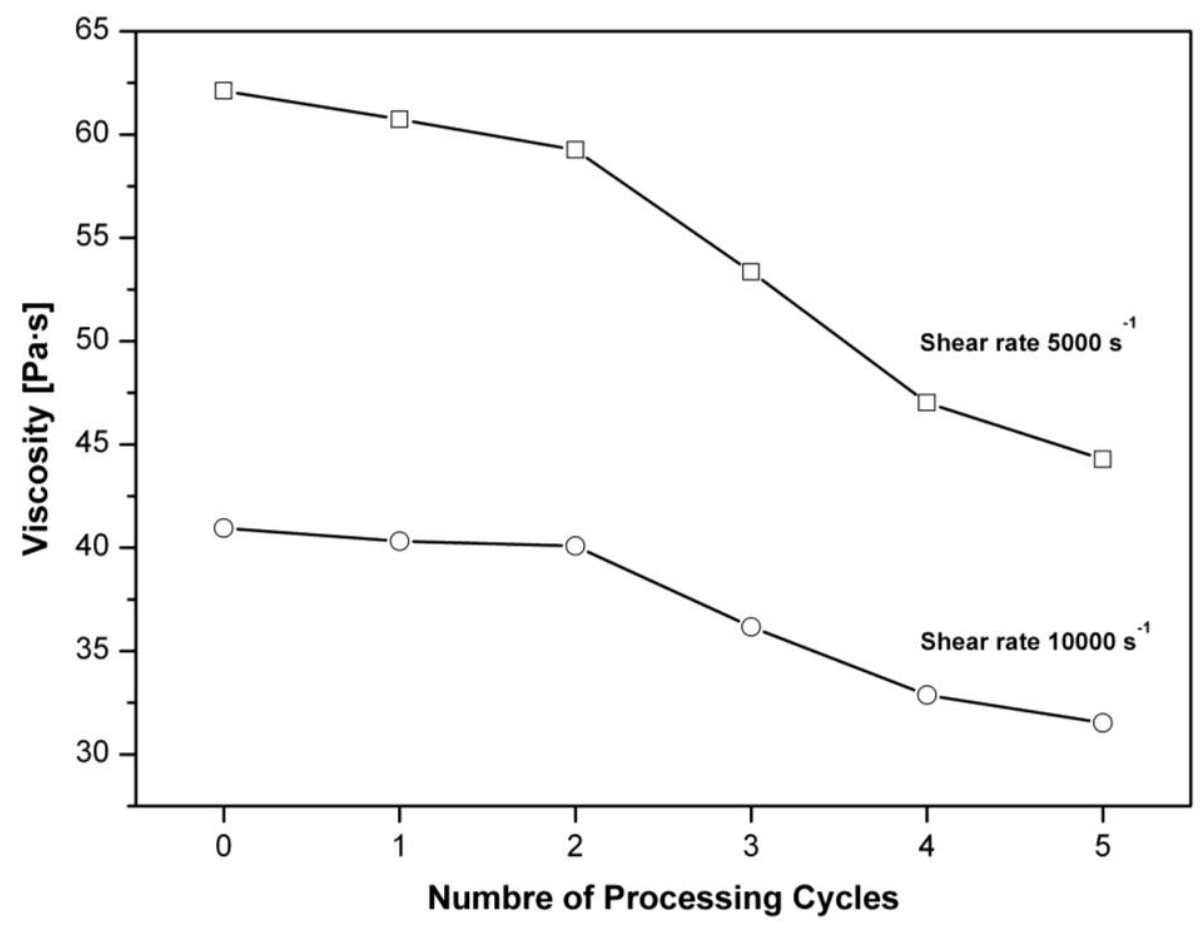

Figure 9. Viscosity of materials versus number of cycles at shear rate 5.000 and $10000 \mathrm{~s}^{-1}$.

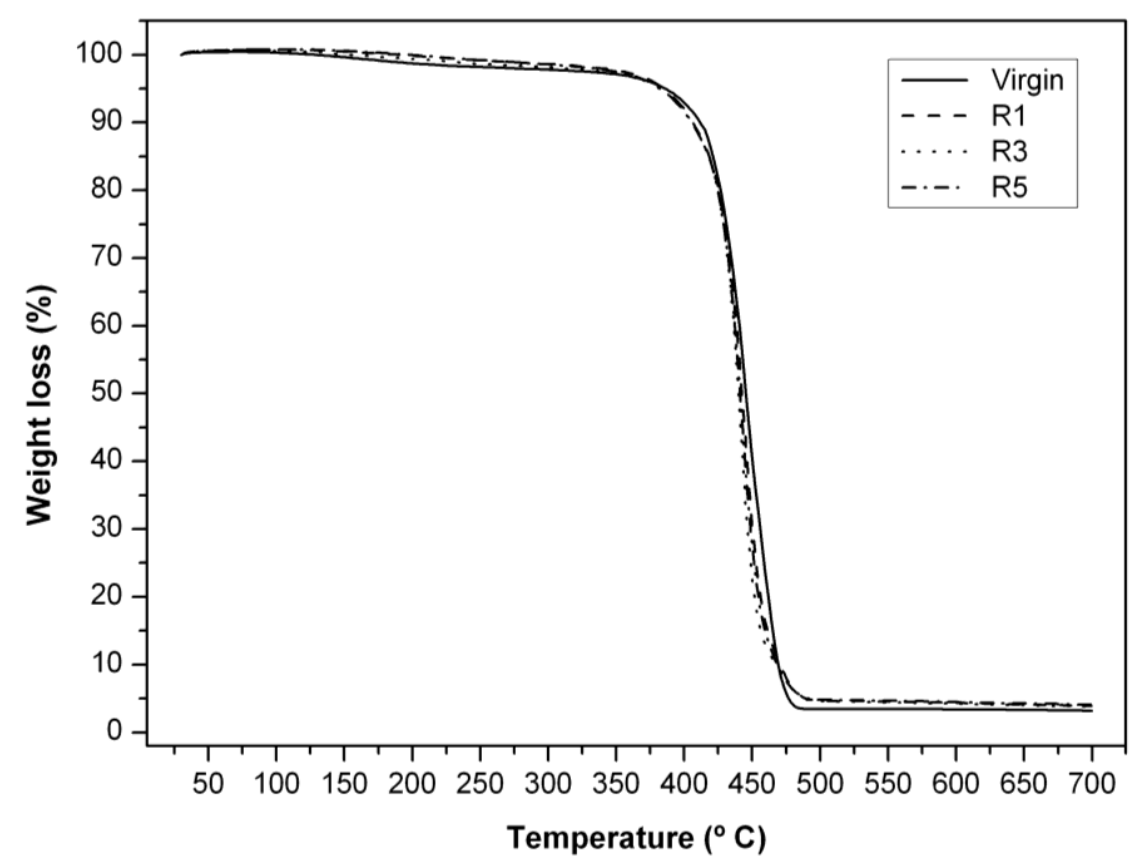

Figure 10. Variation of weight loss in PA6 versus processing cycles. 
Tables

Table 1. Mechanical properties of material virgin and reprocessed PA6.

\begin{tabular}{lcccccc}
\hline Material & V & R1 & R2 & R3 & R4 & R5 \\
\hline Tensile strength [MPa] & 46.59 & 44.99 & 47.20 & 46.648 & 44.78 & 45.93 \\
Elongation at break [\%] & 207 & 209 & 217 & 216 & 212 & 205 \\
Hardness [Shore D] & 74.07 & 73.27 & 73.07 & 72.83 & 72.87 & 73.00 \\
Charpy Unnotched Impact Strength & 45.09 & 35.77 & 31.75 & 31.15 & 28.70 & 28.61 \\
\hline
\end{tabular}

Table 2. Dependent parameters of the Cross-WLF model for PA6.

\begin{tabular}{ccccccccc}
\hline Material & $\mathbf{n}[-]$ & $\tau^{*}[\mathbf{P a}]$ & $\mathbf{D 1}[\mathbf{P a} \cdot \mathbf{s}]$ & $\mathbf{D 2}\left[{ }^{\mathbf{0}} \mathbf{C}\right]$ & $\begin{array}{c}\mathbf{D 3} \\
{[\mathbf{K} / \mathbf{P a}]}\end{array}$ & $\mathbf{A 1}[-]$ & $\tilde{\mathbf{A}} \mathbf{2}[\mathbf{K}]$ & $\eta_{0}[\mathbf{P a} / \mathbf{s}]$ \\
\hline $\mathbf{V}$ & 0.3551 & 90457 & $1.11791 \cdot 10^{15}$ & 323.15 & 0 & 35.65 & 51.60 & 745.63 \\
$\mathbf{R 1}$ & 0.3635 & 87007 & $9.80508 \cdot 10^{14}$ & 323.15 & 0 & 35.57 & 51.60 & 696.45 \\
$\mathbf{R 2}$ & 0.3663 & 115504 & $1.37925 \cdot 10^{15}$ & 323.15 & 0 & 36.57 & 51.60 & 446.21 \\
$\mathbf{R 3}$ & 0.3498 & 133992 & $9.87610 \cdot 10^{14}$ & 323.15 & 0 & 36.56 & 51.60 & 322.03 \\
$\mathbf{R 4}$ & 0.3843 & 118250 & $2.10833 \cdot 10^{14}$ & 323.15 & 0 & 34.94 & 51.60 & 245.78 \\
$\mathbf{R 5}$ & 0.4027 & 115452 & $2.07138 \cdot 10^{14}$ & 323.15 & 0 & 35.12 & 51.60 & 209.60 \\
\hline
\end{tabular}

Table 3. Variation of crystallinity compared to reprocessing of the PA6.

\begin{tabular}{|c|c|c|c|c|c|c|}
\hline & $\mathbf{V}$ & R1 & $\mathbf{R 2}$ & $\mathbf{R 3}$ & R4 & $\mathbf{R 5}$ \\
\hline$\overline{\text { Cristallinity }\left(\mathrm{Jg}^{\wedge}-1\right)}$ & 56.05 & 50.15 & 47.20 & 46.6 & 45.78 & 43.93 \\
\hline
\end{tabular}

Table 4. Variation of the inflection point between virgin and reprocessed material.

\begin{tabular}{cccc}
\cline { 2 - 4 } & \multicolumn{3}{c}{ Temperatures $\left({ }^{\mathbf{0}} \mathbf{C}\right)$} \\
\cline { 2 - 4 } & Starting Point & End point & Inflection Point \\
\hline Virgin & 423.83 & 465.64 & 448.65 \\
R1 & 425.02 & 458.17 & 446.24 \\
R2 & 424.08 & 455.34 & 444.85 \\
R3 & 424.92 & 453.64 & 443.27 \\
R4 & 423.87 & 454.67 & 442.43 \\
R5 & 424.58 & 456.27 & 441.18 \\
\hline
\end{tabular}

